\title{
Amplifier and laser of thulium-doped silica optical fiber
}

\section{Amplificador y láser de fibra óptica de sílice dopada con tulio}

\author{
Enar Franco-Rodríguez, Sebastián Jarabo ${ }^{\mathrm{s}^{*}}$, Francisco J. Salgado-Remacha ${ }^{\mathrm{s}}$ \\ Departamento de Física Aplicada, Facultad de Ciencias, Universidad de Zaragoza (Spain) \\ (" E-mail: sjarabo@unizar.es \\ s: SEDOPTICA member
}

Received: 09/02/2020 Accepted: 18/03/2020

DOI: $10.7149 /$ OPA.53.2.51038

\begin{abstract}
:
We present in this work a thulium-doped fiber laser with ring cavity in continuous wave operation. The pumping is based on Erbium-doped fiber lasers at $1600 \mathrm{~nm}$. The operation with continuous wave pumping and pulsed pumping has been studied, obtaining efficiencies of 35\% and $38 \%$ respectively. These efficiencies are higher than the known efficiencies pumping at $1600 \mathrm{~nm}$. The emission spectrum has two peaks located between $1910 \mathrm{~nm}$ and $1920 \mathrm{~nm}$. As the same time, we present measurements of the spectral gain of the thulium-doped fiber. As an example, we have implemented a thulium-doped fiber amplifier, demonstrating to be able to amplify a supercontinuum spectrum in the interval between $1800 \mathrm{~nm}$ and $2000 \mathrm{~nm}$, which could be used to pump highly nonlinear fibers with a zero-dispersion wavelength in this region.
\end{abstract}

Key words: Thulium-Doped Fiber, Laser, Optical Amplifier, Supercontinuum Spectrum

\section{RESUMEN:}

En este trabajo se presenta un láser de fibra dopada con tulio en cavidad de anillo, operando en modo continuo. El bombeo se realiza en la zona de $1600 \mathrm{~nm}$ mediante láseres de fibra dopada con erbio. Se ha estudiado el comportamiento con bombeo en modo continuo y en modo pulsado, obteniendo unas eficiencias del 35\% y del 38\%, respectivamente. Estas eficiencias son superiores a las típicas que se consiguen bombeando en $1600 \mathrm{~nm}$. El láser emite en dos longitudes de onda separadas unos pocos nanómetros, situadas entre $1910 \mathrm{~nm}$ y $1920 \mathrm{~nm}$. Al mismo tiempo, se presentan también medidas de ganancia espectral de la fibra dopada con tulio. A modo de ejemplo, se ha realizado un amplificador de fibra dopada con tulio y se ha demostrado su viabilidad amplificando un espectro supercontinuo en la región comprendida entre $1800 \mathrm{~nm}$ y $2000 \mathrm{~nm}$, el cual podría utilizarse para bombear fibras no lineales cuya longitud de onda de dispersión cero esté situada en dicha región espectral.

Palabras clave: Fibra Dopada con Tulio; Láser; Amplificador Óptico; Espectro Supercontinuo

\section{REFERENCES AND LINKS / REFERENCIAS Y ENLACES}

[1] K. Scholle, S. Lamrini, P. Koopmann, P. Fuhrberg, " $2 \mu \mathrm{m}$ Laser Sources and Their Possible Applications", Frontiers in Guided Wave Optics and Optoelectronics, Bishnu Pal (Ed.), InTech (2010).

[2] A. E. Budarnykh, A. D. Vladimirskaya, I. A. Lobach, S. I. Kablukov, "Broad-range self-sweeping singlefrequency linearly polarized Tm-doped fiber laser," Opt Lett 43, 5307-5310 (2018).

[3] R. Liao, Y. Song, W. Liu, H. Shi, L. Chai, M. Hu, "Dual-comb spectroscopy with a single free-running thulium-doped fiber laser," Opt Express 26, 11046-11054 (2018).

[4] A. Pal, R. Sen, K. Bremer, S. Yao, E. Lewis, T. Sun, K. T. V. Grattan, "'All-fiber" tunable laser in the $2 \mu \mathrm{m}$ region, designed for CO2 detection", App Opt 51, 7011-7015 (2012).

[5] A. M. Heidt, J. H. V. Price, C. Baskiotis, J. S. Feehan, Z. Li, S. U. Alam, D. J. Richardson, "Mid-infrared ZBLAN fiber supercontinuum source using picosecond diode-pumping at $2 \mu \mathrm{m}, "$ Opt Express 21, 24281-24287 (2013). 
[6] S. Liang, L. Xu, Q. Fu, Y. Jung, D. P. Shepherd, D. J. Richardson, S. U. Alam, "295-kW peak power picosecond pulses from a thulium-doped-fiber MOPA and the generation of watt-level $>2.5$-octave supercontinuum extending up to $5 \mu \mathrm{m}$," Opt Express 26, 6490-6498 (2018).

[7] S. D. Jackson, "The spectroscopic and energy transfer characteristics of the rare earth ions used for silicate glass fibre lasers operating in the shortwave infrared," Laser \& Photon Rev 3, 466-482 (2009).

[8] A. Sincore, J. D. Bradford, J. Cook, L. Shah, M. C. Richardson, "High Average Power Thulium-Doped Silica Fiber Lasers: Review of Systems and Concepts," IEEE J Sel Top Quantum Electron 24, 0901808 (2018).

[9] C. Romano, R. E. Tench, J. M. Delavaux, "Simulation of $2 \mu \mathrm{m}$ single clad thulium-doped silica fiber amplifiers by characterization of the $3 \mathrm{~F}_{4}-3 \mathrm{H}_{6}$ transition," Opt Express 26, 26080-26092 (2018).

[10] Y. Ju, C. Yang, B. Yao, T. Dai, X. Duan, Y Ding, "The improvement of output characteristics on Tm3+doped all-fibre laser operating at $1908 \mathrm{~nm}, "$ J Modern Opt 64, 1298-1301 (2017).

[11] J Geng, J. Wu, S. Jiang, J. Yu, "Efficient operation of diode-pumpled single frequency thulium-doped fiber lasers near $2 \mu \mathrm{m}$," Opt Lett 32, 355-357 (2007).

[12] J. Xu, M. Prabhu, J. Lu, K. I. Ueda, D. Xing, "Efficient double-clad thulium-doped fiber laser with a ring cavity," App Opt 40, 1983-1988 (2001).

[13] J. Wang, Z. Quan, H. Shen, X. Chen, Y. Qi, B. He, J. Zhou, "Monolithic Tm-doped single-mode fiber oscillator with 27.1 W output power at $1.94 \mu \mathrm{m}$," Laser Phys Lett 15, 105103 (2018).

[14] K. Yin, R. Zhu, B. Zhang, G. Liu, P. Zhou, J. Hou, "300 W-level, wavelength-widely-tunable, all-fiber integrated thulium-doped fiber laser," Opt Express 24, 11085-11090 (2016).

[15] J. Wu, Z. Yao, J. Zong, S. Jiang, "Highly efficient high-power thulium-doped germanate glass fiber laser," Opt Lett 32, 638-640 (2006).

[16] Z. Li, S. U. Alam, Y. Jung, A. M. Heidt, D. J. Richardson, "All-fiber, ultra-wideband tunable laser at $2 \mu \mathrm{m}, "$ Opt Lett 38, 4739-4742 (2013).

[17] S. Kharitonov, C. S. Brès, "Isolator-free unidirectional thulium-doped fiber laser," Light: Science \& Applications 4, e340 (2015).

[18] M. Meleshkevich, N. Platonov, D. Gapontsev, A. Drozhzhin, V. Sergeev, V. Gapontsev, "415W singlemode CW thulium fiber laser in all-fiber format, " Proc. Eur. Conf. Lasers Electro-Optics Int. Quantum Electron. Conf. Dig., Munich, Germany, Paper no. CP2_3 (2007).

[19] Z. Li, A. M. Heidt, N. Simakov, Y. Jung, J. M. O. Daniel, S. U. Alam, D. J. Richardson, "Diode-pumped wideband thulium-doped fiber amplifiers for optical communications in the 1800-2050 nm window," Opt Express 21, 26450-26455 (2013).

[20] J. Wang, S. Liang, Q. Kang, Y. Jung, S. U. Alam, D. J. Richardson, "Broadband silica-based thulim doped fiber amplifier employing multi-wavelength pumping," Opt Express 24, 23001- 23008 (2016).

[21] C. Lavieja, S. Jarabo, M. Marín-Doñágueda, Í. J. Sola, "Mode-locked erbium-doped fiber lasers as source for optical sensor networks over C and L bands," Opt Fiber Technol 19, 476-481 (2013).

[22] C. W. Chang, S. Chi, "Mode-locked erbium-doped fibre ring laser using nonlinear polarization rotation," J Modern Opt 45, 355-362 (1998).

[23] J. E. Saldaña Díaz, Generación de supercontinuo mediante fibras ópticas altamente no lineales bombeadas con láseres de fibra pulsados por mode-locking pasivo, Tesis Doctoral, Universidad de Zaragoza (2018).

\section{Introducción}

El desarrollo de fuentes láser en la región espectral de $2 \mu$ m suscita un gran interés debido a sus atractivas aplicaciones en diferentes campos [1]. En el ámbito médico pueden utilizarse en aplicaciones quirúrgicas de precisión en tejidos biológicos, tanto duros como blandos. Por una parte, la profundidad de penetración en los tejidos biológicos es muy baja (algunas décimas de milímetro) debido a la fuerte absorción del agua en esta zona espectral, permitiendo cortar los tejidos de forma muy precisa. El origen de esta fuerte absorción se debe a los niveles de energía vibracionales asociados a los enlaces $\mathrm{OH}$ de las moléculas de agua. Por otra parte, causa una fuerte coagulación que suprime el sangrado en el corte con láser durante los procedimientos quirúrgicos. En procesado de materiales, estos láseres resultan muy apropiados para 
el procesado de materiales plásticos que sean transparentes en el rango visible. La mayoría de los plásticos relevantes poseen una absorción suficientemente alta en torno a las $2 \mu \mathrm{m}$, lo que hace posible cortarlos, marcarlos o soldarlos entre sí con facilidad. Además, no es necesario añadir aditivos a los plásticos para aumentar su absorción [1], como sí ocurre cuando se emplean láseres que emiten en longitudes de onda más bajas. En espectroscopia y detección láser, estos láseres permiten la detección de gases en la atmósfera (por ejemplo, $\mathrm{H}_{2} \mathrm{O}[2,3]$ o $\mathrm{CO}_{2}$ [4]), los cuales presentan líneas de absorción en la región de $2 \mu \mathrm{m}$. Como esta región espectral se considera "eye safe" (la radiación se absorbe antes de que alcance la retina, evitando así que se produzcan daños irreparables), son especialmente indicados para aplicaciones en campo abierto (free space), como comunicaciones ópticas por láser, detección a larga distancia (gases y aerosoles en la atmósfera) y medidas de velocidad de viento mediante LIDAR (LIght Detection And Ranging). Por último, también se utilizan como fuente de excitación de fibras ópticas de materiales fuertemente no lineales, generando un espectro supercontinuo, lo que se emplea para desarrollar fuentes ópticas con un espectro de emisión muy ancho (superior a las 3 micras de anchura). Se han publicado fuentes cuyo espectro se extiende desde los $750 \mathrm{~nm}$ hasta los $4000 \mathrm{~nm}$ y $5000 \mathrm{~nm}$, empleando fibras de ZBLAN (fluoruro de circonio, bario, lantano, aluminio y sodio) [5] y de InF3 (fluoruro de indio) [6], respectivamente.

En la región de $2 \mu \mathrm{m}$, pueden desarrollarse láseres y amplificadores basados en materiales dopados con iones de tulio ( $\mathrm{Tm}_{3+}$ ) o de holmio ( $\mathrm{Ho}_{3}$ ). El ion de tulio puede bombearse con mayor facilidad, bien en 790 $\mathrm{nm}$ o bien en $1650 \mathrm{~nm}$, mientras que el ion de holmio sólo puede excitarse en $1.9 \mu \mathrm{m}$, lo que dificulta su empleo. Además, pueden fabricarse fibras ópticas dopadas con estos iones (con uno de ellos o ambos) para desarrollar amplificadores y láseres en la región de $2 \mu \mathrm{m}$ que sean todo-fibra, lo que permite construir dispositivos compactos, estables y ligeros debido a las ventajas inherentes a la fibra óptica. En este trabajo nos centramos en la fibra óptica de sílice dopada con tulio.

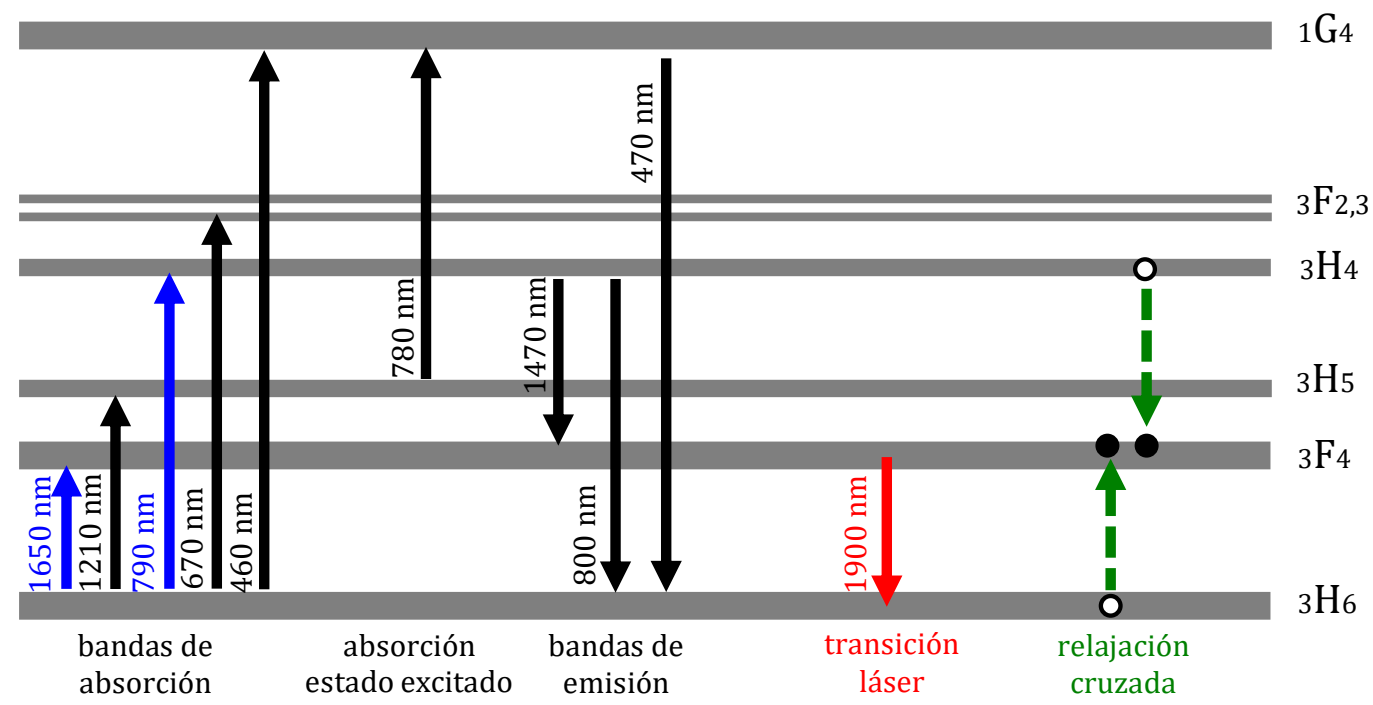

Fig.1. Principales transiciones ópticas del ion de tulio ( $\mathrm{Tm}_{3+}$ ) en sílice. En azul, las bandas de bombeo más utilizadas. En verde, el proceso de transferencia de energía entre iones (relajación cruzada) más relevante.

Para entender correctamente los mecanismos de amplificación y láser es conveniente conocer la espectroscopia del ion de tulio $\left(\mathrm{Tm}_{3+}\right)$ en sílice. En la Fig. 1 se muestran las principales transiciones ópticas para el ion de tulio en sílice $\left(\mathrm{Tm}_{3+}\right)[1,8]$. Debido al efecto Stark, los niveles de energía presentan un gran ensanchamiento, siendo en realidad bandas de energía. De hecho, la transición láser $\left(3 \mathrm{~F}_{4} \rightarrow{ }_{3} \mathrm{H}_{6}\right)$ es de las más anchas que se producen en iones de tierras raras, extendiéndose desde los $1600 \mathrm{~nm}$ hasta los 2200 nm. En la Fig. 2 se muestran los espectros de las secciones eficaces de absorción y de emisión para dicha transición [7], aunque se han publicado espectros con ligeras variaciones dependiendo del autor y de la fibra dopada empleada [8]. La sección eficaz de emisión es máxima en torno a $1850 \mathrm{~nm}$, mientras que la sección eficaz de absorción es máxima en torno a $1650 \mathrm{~nm}$. Esta clara separación entre ambas, de aproximadamente $200 \mathrm{~nm}$, facilita que se produzcan los efectos de amplificación óptica y de láser. Interesa bombear en una longitud de onda para la que la absorción sea lo más alta posible, pero a su vez 
que la emisión estimulada sea lo menor posible. Es decir, en torno a $1620 \mathrm{~nm}$. Otro factor que favorece la inversión de población es que el tiempo de vida media de la transición láser $[8,9]$ es relativamente largo, entre $250 \mu$ s y $650 \mu$ s, debido principalmente a un proceso de decaimiento no radiativo (asistido por fonones). Aunque también hay decaimiento radiativo (emisión espontánea), su influencia es pequeña ya que su tiempo de vida es mucho más largo, entre $4.2 \mathrm{~ms}$ y $6.6 \mathrm{~ms}$.

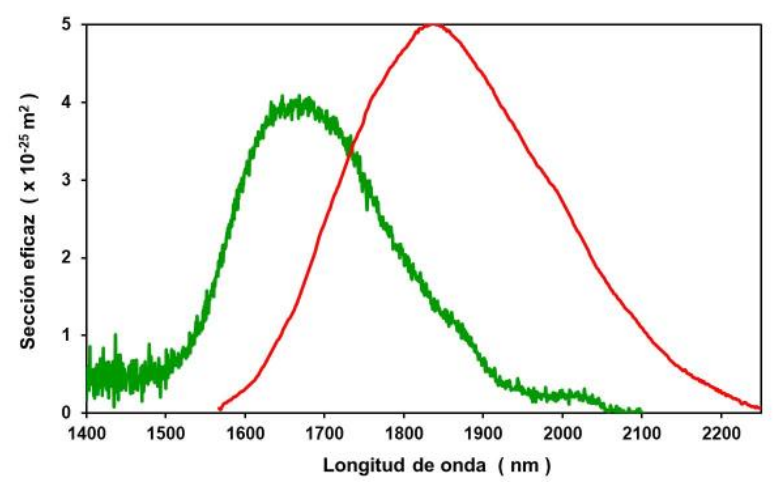

Fig.2. Espectros de las secciones eficaces de absorción (línea verde) y de emisión (línea roja) de la transición láser del ion de tulio ( $\mathrm{Tm}_{3+}$ ) en sílice [7].

Usualmente, las fibras de sílice dopadas con tulio (TDF, thulium-doped fiber) se bombean aprovechando la transición de $1600 \mathrm{~nm}\left({ }_{3} \mathrm{H}_{6} \rightarrow_{3} \mathrm{~F}_{4}\right)$ o la transición de $790 \mathrm{~nm}\left({ }_{3} \mathrm{H}_{6} \rightarrow_{3} \mathrm{H}_{4}\right)$, debido a que se dispone de fuentes de bombeo adecuadas para ambas. Bombeando en $1600 \mathrm{~nm}$, el comportamiento del medio activo puede considerarse como un esquema de 4 niveles, mientras que bombeando en $790 \mathrm{~nm}$, se asocia a un esquema de 3 niveles. La transición de $1600 \mathrm{~nm}$ posee la ventaja de que, además de con láseres de semiconductor, puede bombearse con láseres de fibra óptica dopada con erbio (EDF, erbium-doped fiber). Como son láseres de fibra pueden acoplarse muy fácilmente al amplificador de TDF. Para la transición de 790 nm, la ventaja radica en la existencia de láseres de semiconductor de alta potencia acoplados a fibra óptica, aunque la propagación en la TDF puede no ser monomodo. La transición de $790 \mathrm{~nm}$ ofrece una sección eficaz de absorción entre 0.45 pm2 y 1 pm2, resultando superior a la de la transición de 1600 nm, aunque la transición de $1600 \mathrm{~nm}$ proporciona una eficiencia cuántica 2 veces superior. Por último, hay que tener en cuenta que la inversión de población mediante bombeo en la transición de $790 \mathrm{~nm}$ se ve favorecida por un proceso de relajación cruzada (ver Fig. 1) que puede proporcionar 2 iones en el estado excitado de la transición láser por cada fotón de bombeo [8], debido al cual se consiguen láseres con eficiencia superior al 40\%. Se han publicado bastantes trabajos dónde se obtienen láseres cuya eficiencia se sitúa entre el $30 \%$ y el $40 \%$ [10-12], e incluso más altas, llegando al 57\% [13,14] e incluso al 68\% [15]. En general, bombeando en la transición de $1650 \mathrm{~nm}$ se consiguen láseres con menor eficiencia, del 11\% [16] y del $25 \%$ [17]. No obstante, bombeando con láseres de EDF se ha llegado a conseguir una eficiencia del $60 \%$ [18]. El principal problema es que las fuentes empleadas emiten lejos del máximo de absorción (1550 nm en la Ref. 16, $1600 \mathrm{~nm}$ en la Ref. 17 y $1567 \mathrm{~nm}$ en la Ref. 18). También se encuentra publicado [16] un láser de TDF, sintonizable desde $1820 \mathrm{~nm}$ hasta $2075 \mathrm{~nm}$ (255 nm), emitiendo una potencia máxima de 30 mW con una relación señal-ruido superior a $40 \mathrm{~dB}$. Comercialmente, la empresa Thorlabs oferta un láser sintonizable (modelo LFLTM) desde $1800 \mathrm{~nm}$ hasta $2040 \mathrm{~nm}$ (240 nm) con una potencia máxima de 35 $\mathrm{mW}$. En cuanto a medidas de amplificación, bombeando en la banda de $1600 \mathrm{~nm}$, se llega a obtener una ganancia de $36 \mathrm{~dB}$ para señal débil y de $20 \mathrm{~dB}$ para potencias de señal de $0 \mathrm{dBm}$ desde $1810 \mathrm{~nm}$ hasta $2050 \mathrm{~nm}(240 \mathrm{~nm})[19,20]$.

En este artículo se desarrollan 2 láseres de TDF con cavidad de anillo operando en modo continuo bombeando en la transición de $1650 \mathrm{~nm}$, obteniendo unas eficiencias superiores al 35\%. Las fuentes de bombeo son láseres de EDF basadas en amplificadores comerciales operando en banda L, para que la longitud de onda de bombeo sea cercana a $1620 \mathrm{~nm}$. Las características principales de la TDF (modelo SMTSF-9/125 de Nufern) proporcionadas por el fabricante son las siguientes: diámetro del núcleo $9 \mu \mathrm{m}$, apertura numérica 0.15 , diámetro de campo modal $10.5 \mu \mathrm{m}$ en $2000 \mathrm{~nm}$, longitud de onda de corte 1750 $\mathrm{nm}$, coeficiente de absorción $27 \mathrm{~dB} / \mathrm{m}$ en $793 \mathrm{~nm}$ y $9 \pm 2 \mathrm{~dB} / \mathrm{m}$ en $1180 \mathrm{~nm}$. En la Fig. 3 se representa el espectro de su coeficiente de atenuación, el cual fue medido con una muestra de $2 \mathrm{~m}$ de longitud 
empleando una fuente de supercontinuo (ver Anexo). Se aprecian claramente las bandas de absorción de $1200 \mathrm{~nm}$ y de $1650 \mathrm{~nm}$. En $1180 \mathrm{~nm}$, el coeficiente de atenuación es $10 \mathrm{~dB} / \mathrm{m}$, en concordancia con el valor proporcionado por el fabricante. Para la banda de $1650 \mathrm{~nm}$, el coeficiente de atenuación presenta un valor máximo de $24 \mathrm{~dB} / \mathrm{m}$. Por tanto, para desarrollar un láser es suficiente con emplear 1 ó 2 metros de TDF. Comparando los valores de la Fig. 3 con los de la Fig. 2 y calculando que la proporción de potencia acoplada en el núcleo de la fibra dopada para $1650 \mathrm{~nm}$ es del 85\%, se estima una concentración de iones de tulio de $1.65 \times 1025 \mathrm{~m}-3$ (750 ppm).

El punto de partida de este artículo es un Trabajo Fin de Grado (de Física) titulado "Láser de fibra óptica dopada con tulio" realizado por Enar Franco Rodríguez en la Universidad de Zaragoza durante el curso académico 2016-2017, del cual se presentan sus resultados más relevantes. Consideramos que estos resultados pueden servir de utilidad para el desarrollo de prácticas docentes de grado o de máster sobre esta temática u otras similares.

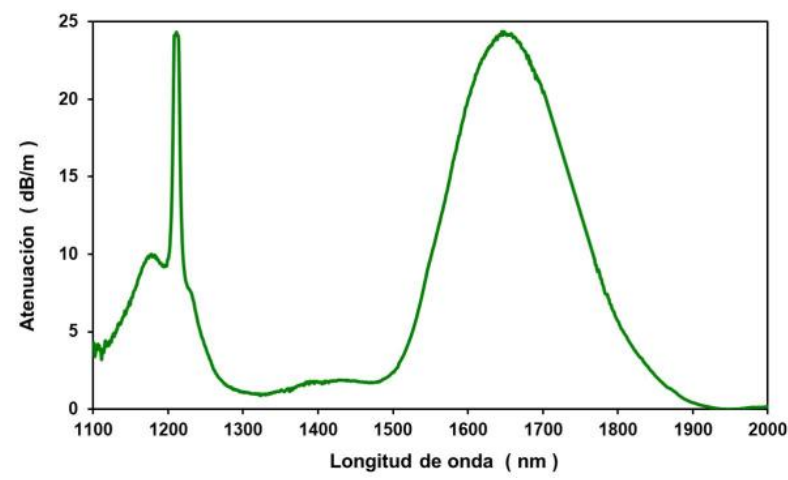

Fig.3. Coeficiente espectral de absorción de la fibra dopada con tulio (modelo SM-TSF-9/125 de Nufern).

\section{Montajes experimentales}

En esta sección se exponen los diferentes montajes experimentales desarrollados para la realización de este trabajo: dos etapas de bombeo, una de ellas en modo continuo y la otra en modo pulsado mediante mode-locking (encadenado de modos), la cavidad en anillo del láser de TDF y el amplificador de TDF.

\section{2.a. Etapa de bombeo operando en modo continuo}

El esquema de esta etapa se muestra en la Fig. 4. Consiste en un láser de EDF con cavidad de anillo, cuya potencia de salida se amplifica mediante un amplificador de fibra óptica dopada con erbio (EDFA, erbiumdoped fiber amplifier) que opera en banda $\mathrm{L}$ y tiene una potencia de salida de saturación de $26 \mathrm{dBm}$ (Manlight, modelo HWT-EDFA-GM-SC-BO-L26; en adelante, EDFA-L26). Como medio activo para el láser de anillo se emplea otro EDFA que también opera en banda L y que tiene una potencia de salida de saturación de $20 \mathrm{dBm}$ (Keopsys, modelo KPS-BT2-L-20-PB-FA; en adelante, EDFA-L20). La potencia láser se extrae de la cavidad de anillo mediante un acoplador 90/10, de forma que se extrae el $90 \%$ de la potencia confinada. Para seleccionar la longitud de onda de emisión del láser, dentro de la cavidad se incluyen un circulador óptico y una red de Bragg en fibra (FBG), cuyo máximo de reflexión se produce para una longitud de onda de $1612.81 \mathrm{~nm}$ (Technica S. A.; anchura espectral de $0.25 \mathrm{~nm}$ a $-3 \mathrm{~dB}$ del máximo). Esta configuración ayuda a maximizar la potencia de salida, aumentando la potencia disponible para bombear la TDF. La potencia emitida por el láser de bombeo alcanza los $12 \mathrm{dBm}$, la cual aumenta hasta los $19.3 \mathrm{dBm}$ con el EDFA-L26. El espectro normalizado a altura unidad de la potencia de bombeo emitida, medida con una resolución de $0.06 \mathrm{~nm}$, se muestra en la Fig. 5. La etapa de bombeo presenta emisión máxima en $1612.7 \mathrm{~nm}$ y la relación señal-ruido en torno a dicha longitud de onda supera los 50 dB. Aunque el segundo amplificador añade en banda $C$ una potencia notable debida a emisión espontánea amplificada, ésta no causa ningún problema ya que también contribuirá, aunque ligeramente, a bombear la TDF. 


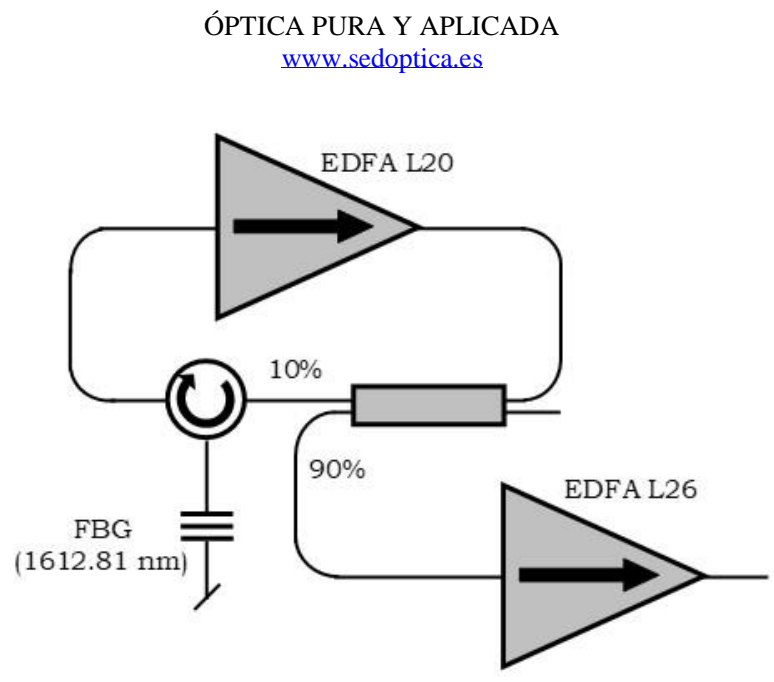

Fig.4. Esquema de la etapa de bombeo operando en modo continuo.

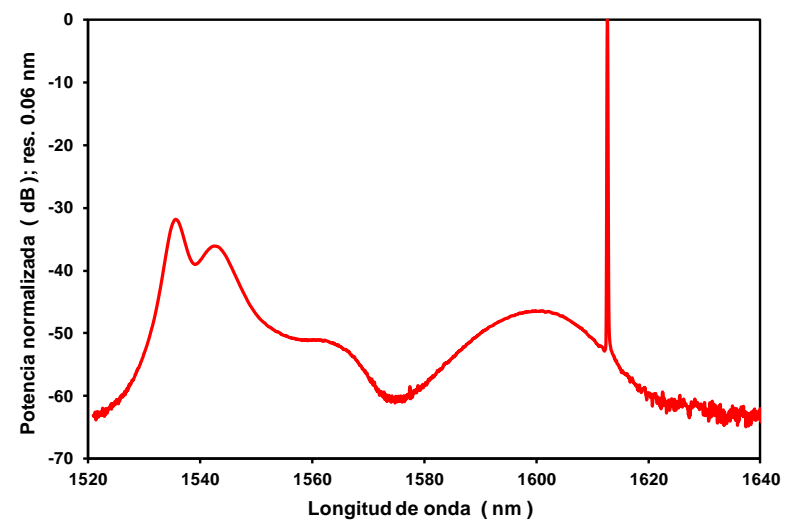

Fig.5. Espectro de potencia emitido por la etapa de bombeo operando en modo continuo.

\section{2.b. Etapa de bombeo operando en modo pulsado}

El esquema de esta etapa se muestra en la Fig. 6. Está formada por un láser de anillo de EDF pulsado mediante mode-locking y un segundo amplificador de EDF (EDFA-L26) para elevar la potencia de los pulsos láser generados. La cavidad láser está construida con el amplificador EDFA-L20, un acoplador 90/10 y el modulador para establecer la condición de mode-locking pasivo, el cual consta de un polarizador lineal (Thorlabs, modelo ILP1550SM) entre dos controladores de polarización (General Photonics, modelo Polarite). Su funcionamiento está basado en el efecto de rotación no lineal de la polarización (NPR, nonlinear rotation polarization), el cual es un fenómeno no lineal que induce una rotación del estado de polarización proporcional a la intensidad de luz y a la longitud del material. En láseres de fibra óptica con cavidad de anillo es una técnica pasiva ampliamente utilizada para conseguir mode-locking [21, 22], ya que las cavidades son muy largas y en las que resulta fácil minimizar las pérdidas, consiguiendo aumentar por tanto la potencia confinada y la magnitud de los efectos no lineales. Ajustando los controladores de polarización se consigue que, inicialmente, tras recorrer la cavidad, el estado de polarización que llega al polarizador lineal sea aproximadamente ortogonal a su dirección de polarización (condición de modulador cerrado). Ahora bien, cuando la potencia es suficientemente elevada (pulsos), el efecto NPR consigue que la potencia llegue al polarizador lineal con un estado de polarización paralelo a su dirección de polarización (condición de modulador abierto) y el láser emite un pulso. Una parte de la potencia del pulso se queda confinada en la cavidad y, tras dar una vuelta a la cavidad, se amplifica y nuevamente encuentra el modulador abierto y se vuelve a emitir otro pulso. La iteración de este proceso origina el tren de pulsos. Para ayudar al efecto no lineal se eligió una relación de acoplo 90/10 para confinar la mayor potencia posible dentro de la cavidad. 


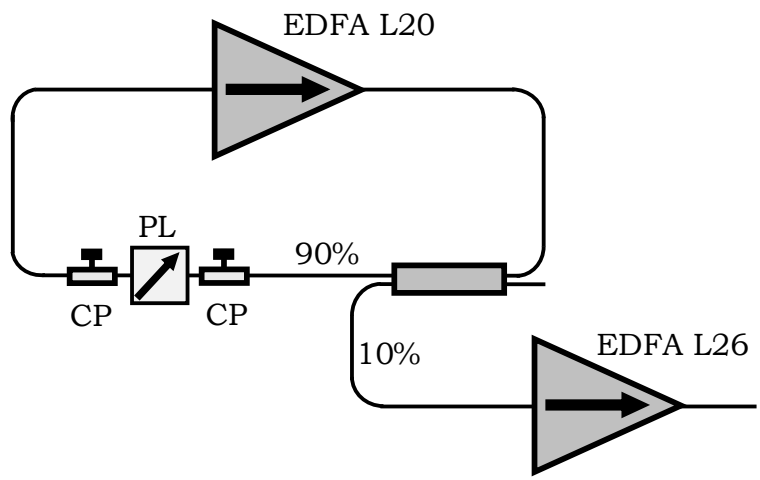

Fig.6. Esquema de la etapa de bombeo operando en modo pulsado.

En la Fig. 7 se muestra el espectro de salida del láser pulsado y el tren de pulsos generado. La frecuencia del tren de pulsos es de $3.27 \mathrm{MHz}$, lo que equivale a que la longitud de la cavidad es aproximadamente de $63 \mathrm{~m}$. El espectro está centrado en $1605 \mathrm{~nm}$. Su anchura espectral a 1/e del máximo es $24 \mathrm{~nm}$, por lo que la duración temporal de los pulsos (a 1/e del máximo) está limitada por transformada de Fourier a 0.228 ps. De la medida del tren de pulsos de la Fig. 7 no es posible determinar la anchura temporal de los pulsos ya que la respuesta temporal del detector empleado no es lo suficientemente rápida. No obstante, mediante la técnica interferométrica de autocorrelación de segundo orden se estimó una duración de 0.33 ps, compatible con el límite establecido por transformada. Para ello se empleó un autocorrelador construido en nuestro laboratorio formado por un interferómetro Mach-Zehnder, un cristal no lineal de BBO $\left(\beta-\mathrm{BaB}_{2} \mathrm{O}_{4}\right)$ y un detector lento (fotodiodo de silicio). El interferograma de la medida de autocorrelación se muestra en la Fig. 8.

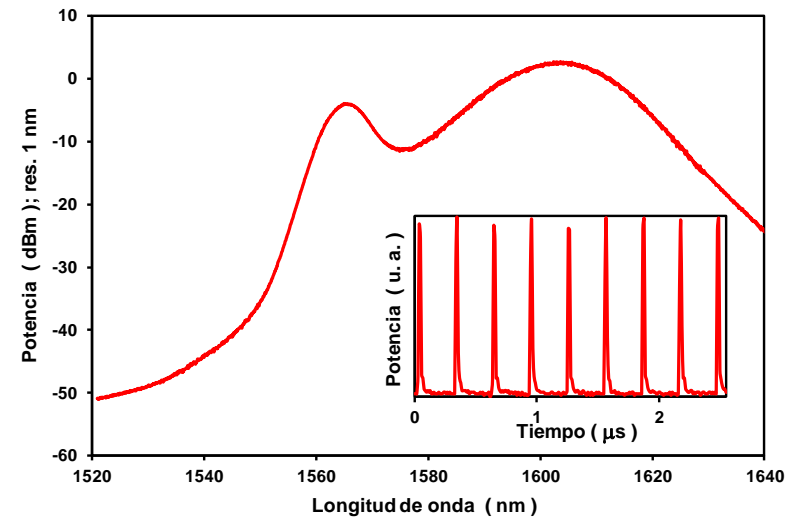

Fig.7. Espectro de potencia emitido por la etapa de bombeo operando en modo pulsado. La frecuencia del tren de pulsos es $3.27 \mathrm{MHz}$.

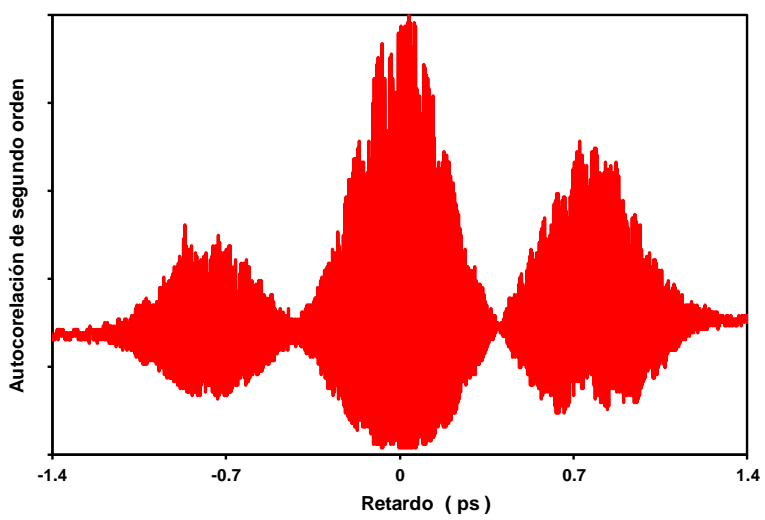

Fig.8. Interferograma de la autocorrelación de segundo orden del tren de pulsos, cuya anchura temporal se estima en 0.33 ps. 


\section{2.c. Cavidad del láser de fibra óptica dopada con tulio}

Tal como se ilustra en la Fig. 9, la cavidad del láser de TDF tiene estructura de anillo. La potencia de bombeo procede de las dos etapas de bombeo descritas en las secciones 2.a (operando en modo continuo) y 2.b (operando en modo pulsado). Como medio activo se usa una muestra de TDF de $1.8 \mathrm{~m}$ de longitud. Esta longitud fue elegida de forma que optimizase la potencia de salida del láser para la máxima potencia de bombeo disponible, tras ensayar 5 longitudes de fibra diferentes (1.4 m, $1.8 \mathrm{~m}, 2 \mathrm{~m}, 4 \mathrm{~m}$ y $6 \mathrm{~m}$ ). Para acoplar de forma eficiente el bombeo se emplea un acoplador WDM 1600/2000. Una de sus entradas presenta alta transmisión hasta $1800 \mathrm{~nm}$, mientras que la otra entrada ofrece alta transmisión desde 1800 $\mathrm{nm}$ en adelante, acoplando nuevamente en el anillo la potencia correspondiente a la zona espectral de amplificación del tulio. Así se optimiza el bombeo de la TDF mientras que a su vez se minimizan las pérdidas de la cavidad. En la cavidad se incluye un aislador óptico (aislamiento de $35 \mathrm{~dB}$ ) para impedir la recirculación de la luz en uno de los sentidos evitando inestabilidades e impidiendo que la fluorescencia contrapropagante sea amplificada. Por último, se utiliza un acoplador 90/10 para extraer el $10 \%$ de la potencia confinada en la cavidad. Con esta relación de acoplo se consigue que las pérdidas de la cavidad no crezcan en exceso y que, por tanto, la potencia de bombeo umbral sea bastante baja ya que no disponemos de demasiada potencia de bombeo. No obstante, hay que tener presente que podría optimizarse la eficiencia del láser modificando la relación de acoplo.

En la Fig. 10 se muestran los factores espectrales de transmisión de ambas entradas del WDM y del aislador, los cuales han sido medidos con la fuente de espectro supercontinuo citada anteriormente (ver anexo). A partir de ellos, se aprecia un buen acoplamiento del bombeo (pérdidas en torno a $0.2 \mathrm{~dB}$ ) y que la cavidad presenta menores pérdidas en la región espectral comprendida entre $1825 \mathrm{~nm}$ y $1950 \mathrm{~nm}$, aproximadamente. Las pérdidas de la cavidad en dicha región espectral son bajas ( $2.9 \mathrm{~dB})$. Teniendo en cuenta además los espectros de absorción y emisión del tulio (Fig. 2) es de esperar que el láser emita en torno a $1900 \mathrm{~nm}$. Es necesario apuntar que en la cavidad no se incluye ningún dispositivo que seleccione la longitud de onda de emisión del láser.

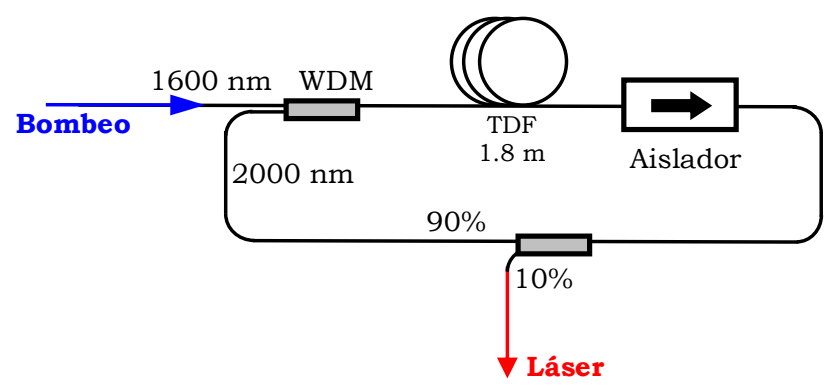

Fig.9. Esquema de la cavidad del láser de fibra óptica dopada con tulio.

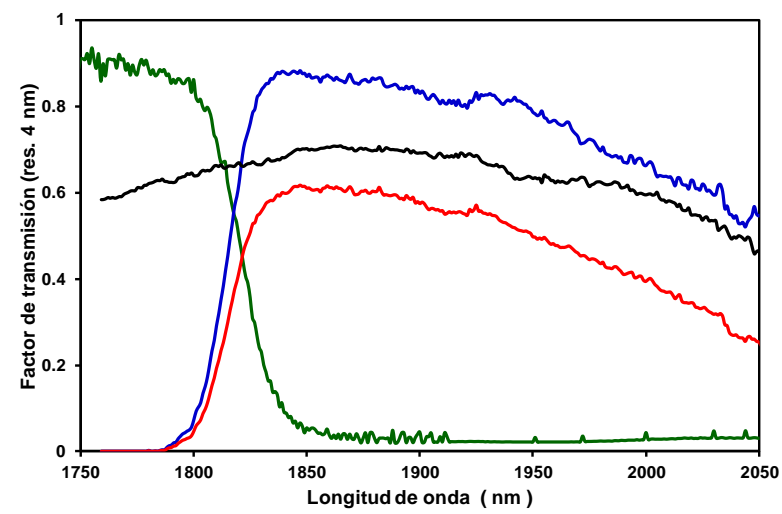

Fig.10. Factor espectral de acoplo del bombeo a la cavidad láser (línea verde). Factores espectrales de transmisión del WDM (línea azul), del aislador (línea negra) y de ambos elementos juntos (línea roja). 


\section{2.d. Amplificador de fibra óptica dopada con tulio}

El esquema del amplificador de TDF (Fig. 11) desarrollado en este trabajo consiste básicamente en abrir la cavidad láser en anillo descrita en la sección 2.c eliminando el acoplador 90/10. El acoplamiento de bombeo no sufre ningún cambio. La otra entrada al acoplador WDM se emplea ahora como entrada para la potencia que se desea amplificar (señal) y el final del aislador será la salida para la potencia amplificada. Como señal se emplea la potencia de la fuente de espectro supercontinuo (ver anexo). La longitud de TDF es ahora de $1.36 \mathrm{~m}$. El aislador óptico debe mantenerse en la instalación para evitar la aparición de efectos de auto-oscilación (o láser accidental).

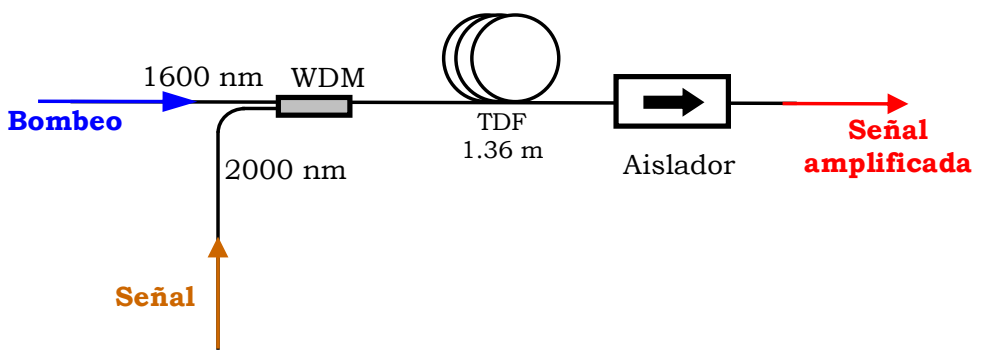

Fig.11. Esquema del amplificador de fibra óptica dopada con tulio.

\section{Resultados}

En esta sección se recogen los resultados más relevantes conseguidos con el láser de TDF empleando los dos láseres de bombeo desarrollados, obteniéndolos a partir de la medida de su recta característica y de la medida del espectro de la potencia emitida. Además, se exponen medidas de ganancia espectral de la TDF.

\section{3.a. Láseres de fibra óptica dopada con tulio}

Los dos láseres de TDF analizados están formados por la cavidad láser en configuración de anillo (sección 2.c) junto con cada una de las etapas de bombeo (en modo continuo, sección 2.a; en modo pulsado, sección 2.b).

En la Fig. 12 se representa la potencia láser emitida en función de la potencia de bombeo empleada (recta característica). Bombeado en modo pulsado, la eficiencia es del $35 \%$ y la potencia de bombeo umbral es $100 \mathrm{~mW}$. Como se esperaba, el comportamiento es lineal para potencias de bombeo superiores a la potencia umbral. Bombeado en modo continuo, la potencia de bombeo umbral es $138 \mathrm{~mW}$ y la eficiencia del láser es del 38\%. Sin embargo, el comportamiento se aleja del funcionamiento lineal. Además, para potencias de bombeo en torno a $200 \mathrm{~mW}$, la potencia de salida era ligeramente inestable, por lo que no se representan sus valores.

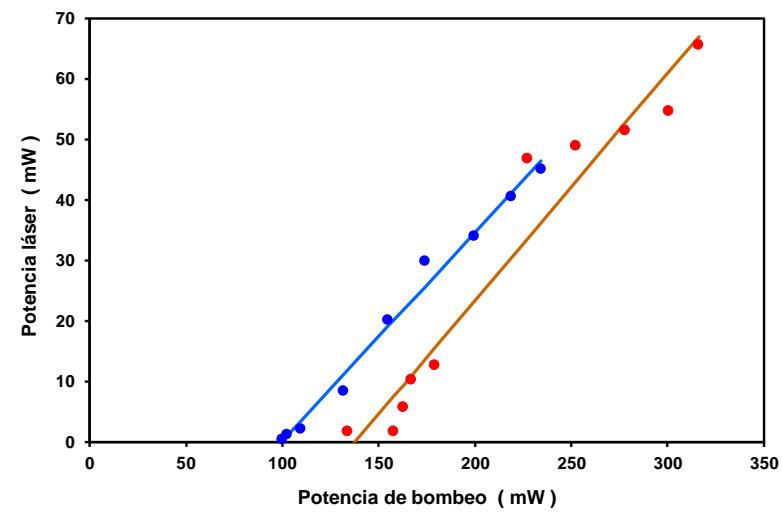

Fig.12. Recta característica del láser de fibra dopada con tulio bombeado en modo continuo (puntos rojos; eficiencia: $38 \%$; potencia de bombeo umbral: $138 \mathrm{~mW}$ ) y en modo pulsado (puntos azules; eficiencia: 35\%; potencia de bombeo umbral: $100 \mathrm{~mW}$ ). 


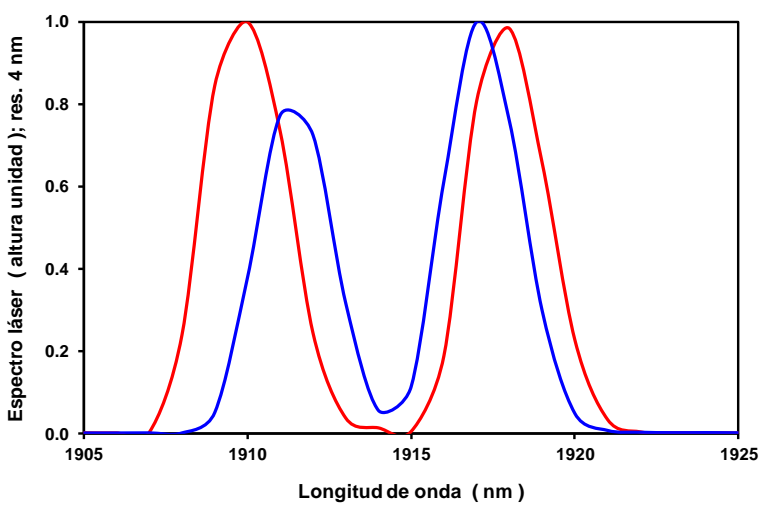

Fig.13. Ejemplo del espectro de emisión del láser de fibra dopada con tulio normalizado a altura unidad (línea roja: potencia de bombeo de $252 \mathrm{~mW}$ en modo continuo; línea azul: bombeo de $174 \mathrm{~mW}$ en modo pulsado).

En la Fig. 13 se muestra un ejemplo de los espectros de emisión de los láseres de TDF. Hay que tener en cuenta que la resolución espectral de estas medidas no es suficiente para determinar la anchura real de estos picos. Ambos láseres emiten en una única longitud de onda para potencias de bombeo bajas. Sin embargo, a partir de una determinada potencia ( $163 \mathrm{~mW}$ para bombeo en modo continuo y $155 \mathrm{~mW}$ para bombeo en modo pulsado) emiten en 2 longitudes de onda relativamente próximas. En la Fig. 14 se muestra la dependencia de estos picos de emisión con la potencia de bombeo. Estas longitudes de onda de emisión corresponden con aquellas para las que la ganancia de la fibra dopada compensa las pérdidas de la cavidad. Por tanto, están determinadas por el perfil espectral de ganancia de la fibra dopada. Hay que tener en cuenta que en la cavidad no se ha incluido ningún filtro que fije la longitud de onda de emisión del láser. Con bombeo en modo continuo ambas longitudes de onda están separadas entre $4 \mathrm{~nm}$ y $9 \mathrm{~nm}$. Las longitudes de onda están comprendidas entre $1907 \mathrm{~nm}$ y $1921 \mathrm{~nm}$. La longitud de onda central aumenta con el bombeo hasta $228 \mathrm{~mW}$, decreciendo para bombeos más altos. No obstante, mientras que una de ellas se desplaza notablemente, la otra se mantiene estable (en torno a $1912 \mathrm{~nm}$ ) para la mayoría de las potencias de bombeo. Con bombeo pulsado la separación entre ambas longitudes de onda es prácticamente constante, de unos $4 \mathrm{~nm}$. Las longitudes de onda están comprendidas entre $1911 \mathrm{~nm}$ y $1919 \mathrm{~nm}$. La longitud de onda central decrece con el bombeo.

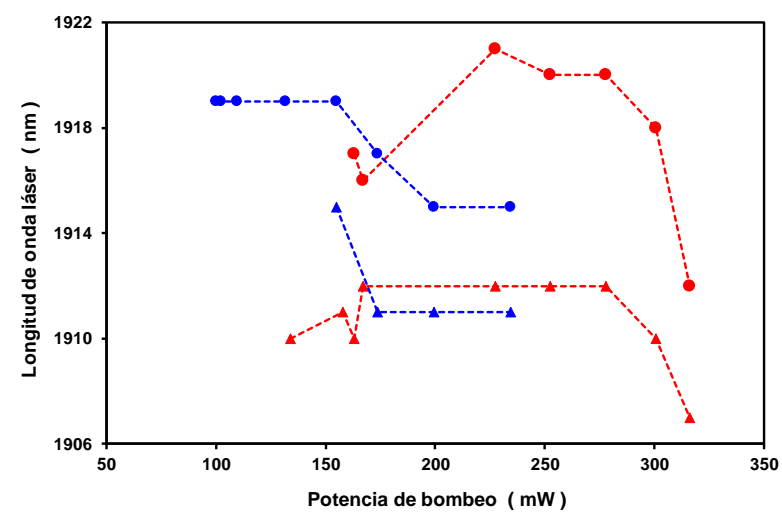

Fig.14. Variación de las longitudes de onda de emisión del láser de fibra dopada con tulio en función de la potencia de bombeo (color rojo: bombeado en modo continuo; color azul: bombeado en modo pulsado).

\section{3.b. Amplificador de fibra dopada con tulio}

Empleando como fuente de señal la de espectro supercontinuo y bombeando con la etapa que opera en modo continuo (sección 2.a), se midió la ganancia espectral del amplificador de TDF. En la Fig. 15 se recogen los espectros de ganancia para diferentes potencias de bombeo. La potencia de bombeo umbral es $109 \mathrm{~mW}$. Para la máxima potencia bombeo $(316 \mathrm{~mW})$ se consigue amplificación en una región espectral con una anchura de $195 \mathrm{~nm}$, concretamente desde $1805 \mathrm{~nm}$ hasta $2000 \mathrm{~nm}$. La máxima ganancia (10.2 dB) se obtiene en torno a $1855 \mathrm{~nm}$. Además, se incluye la medida de la atenuación del amplificador cuando no 
se acopla bombeo a la fibra dopada. Conforme aumenta la potencia de bombeo, la ganancia aumenta, su espectro se ensancha y la longitud de onda de máxima ganancia disminuye desde $1892 \mathrm{~nm}$ hasta $1855 \mathrm{~nm}$. Los perfiles espectrales de amplificación son bastante planos en la zona de mayor ganancia, lo que justifica que el láser pueda emitir en varias zonas espectrales simultáneamente, siempre que la ganancia compense las pérdidas de la cavidad en varias longitudes de onda. Si construimos un láser cerrando la cavidad con una relación de acoplo alta (pérdidas de la cavidad bajas), la longitud de onda del láser tendería hacia la zona de $1930 \mathrm{~nm}$. Disminuyendo la relación de acoplo, aumentaríamos las pérdidas de la cavidad y la longitud de onda de emisión del láser iría disminuyendo progresivamente hacia la región de $1825 \mathrm{~nm}$. No es posible ser más precisos porque, como la potencia confinada en la cavidad es mucho mayor que la potencia empleada para medir la ganancia del amplificador, el perfil de ganancia en el láser se desplazará hacia longitudes de onda más altas, como es habitual en los medios activos cuyo comportamiento puede asociarse con esquemas de dos bandas. No obstante, estas curvas de ganancia nos permiten estimar para nuestra cavidad láser los valores de la potencia de bombeo umbral y de la longitud de onda de emisión en función de la relación de acoplo. También justifican que la longitud de onda de emisión láser disminuya ligeramente al aumentar la potencia de bombeo, siempre que asumamos que la transición láser esté afectada por mecanismos de ensanchamiento de tipo inhomogéneo.

Teniendo en cuenta los factores espectrales de transmisión del aislador y del acoplador WDM se determina también la ganancia espectral de la TDF, obteniendo los espectros de la Fig. 16. El bombeo umbral es $87 \mathrm{~mW}$ y, para máximo bombeo, la ganancia es máxima en $1835 \mathrm{~nm}$, alcanzando los $12.8 \mathrm{~dB}$. Conforme disminuye la potencia de bombeo, la longitud de onda de máxima ganancia disminuye progresivamente hasta $1898 \mathrm{~nm}$. Estas medidas nos permiten estimar que, si los elementos que componen la cavidad fuesen ideales, podría conseguirse amplificación en un rango espectral más ancho, que podría superar los $300 \mathrm{~nm}$ de anchura. Esta anchura sería el límite para el rango de sintonía de un láser en el que incluyamos algún elemento selector de longitud de onda.

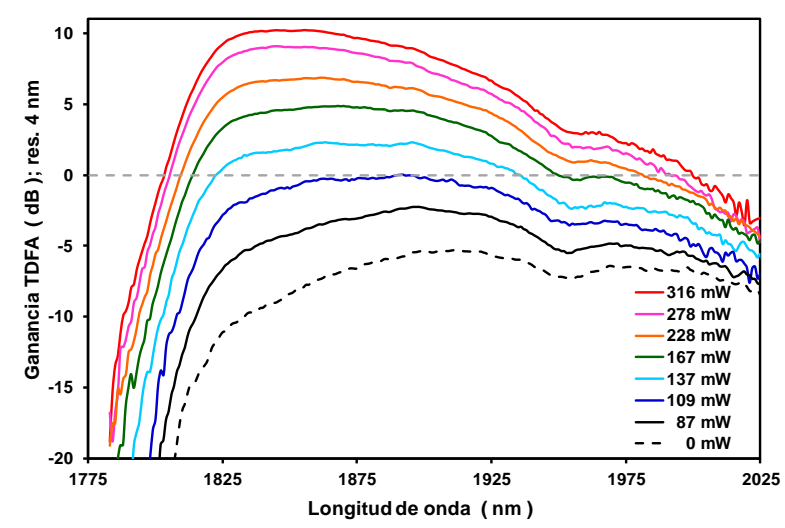

Fig.15. Ganancia espectral del amplificador de fibra óptica dopada con tulio para varias potencias de bombeo.

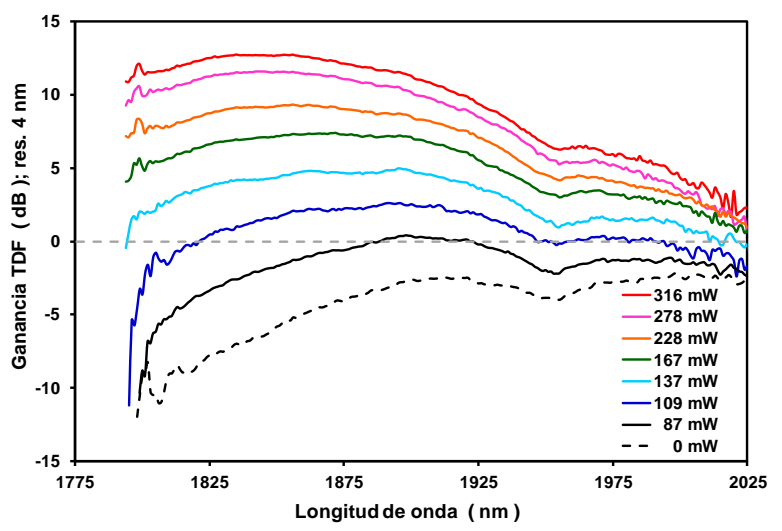

Fig.16. Ganancia espectral de la fibra óptica dopada con tulio para varias potencias de bombeo. 


\section{3.c. Fuente de supercontinuo con espectro mejorado en la región de $\mathbf{2}$ micras}

Con el amplificador de TDF es posible mejorar el espectro de la fuente de supercontinuo (ver anexo) en la región de 2 micras, tal como se muestra en la Fig. 17. Aunque su espectro es considerablemente más estrecho que el de la fuente original, la nueva fuente mejora la potencia desde $1803 \mathrm{~nm}$ hasta $2000 \mathrm{~nm}$, alcanzando los $3.2 \mathrm{dBm} / \mathrm{nm}$ en $1845 \mathrm{~nm}$. Así pues, resulta suficientemente ancha para muchas aplicaciones y, además, tiene una potencia de $207 \mathrm{~mW}$ en dicha región espectral, siendo 5.7 veces mayor que la potencia de la fuente original. Como la fuente original está pulsada (tren de pulsos a $1.4 \mathrm{MHz}$ con una anchura temporal de $0.2 \mathrm{ps}$ ), la nueva fuente también lo estará y, si suponemos que la duración de los pulsos amplificados no cambia, la potencia de pico de los pulsos amplificados puede estimarse en 7 $\mathrm{KW} / \mathrm{nm}$ en $1845 \mathrm{~nm}$ y de $0.7 \mathrm{MW} / \mathrm{nm}$ integrando todo el espectro.

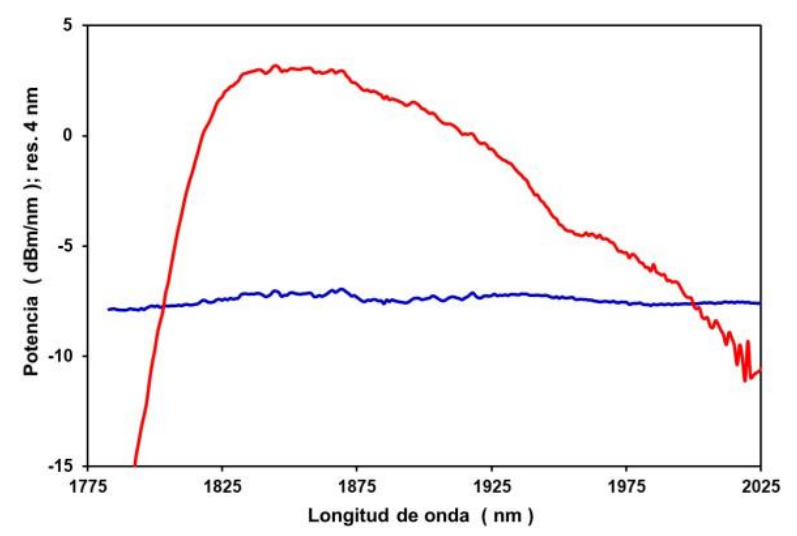

Fig.17. Espectros supercontinuos de la fuente original (línea azul) y de la fuente amplificada mediante la fibra dopada con tulio (línea roja).

\section{Conclusiones}

En este trabajo se ha montado un láser de TDF con cavidad de anillo para operación en modo continuo. El bombeo de la TDF se ha llevado a cabo con láseres de EDF para aprovechar la transición de bombeo en torno a $1600 \mathrm{~nm}$, bien operando en modo continuo o bien operando en modo pulsado. Tras medir la potencia láser emitida en función de la potencia de bombeo se ha determinado su eficiencia y su potencia de bombeo umbral. Bombeando en modo pulsado, la eficiencia es del $35 \%$, mientras que, bombeando en modo continuo, la eficiencia es ligeramente superior, alcanzando valores del $38 \%$. Las ligeras diferencias de comportamiento son debidas a que cada fuente de bombeo presenta un espectro diferente y aprovecha de forma distinta los perfiles de absorción y de emisión de la TDF. El modo de operación del bombeo, continuo o pulsado, apenas influye debido a que el tiempo de vida media del ion de tulio es mucho más largo que las dinámicas que aparecen en el tren de pulsos de bombeo. Bombeando en modo continuo, se dispone de mayor potencia de bombeo y se alcanza una potencia de salida cercana a los $70 \mathrm{~mW}$, resultando ser más que suficiente para muchas aplicaciones. Comparando con la bibliografía, las eficiencias son similares a las típicas que se obtienen habitualmente bombeando al tulio en la banda de $790 \mathrm{~nm}$ (entre el $30 \%$ y el $40 \%$ ) y superiores a las habituales bombeando en la banda de $1600 \mathrm{~nm}$ (entre el $10 \%$ y el $25 \%$ ). No obstante, están lejos de las máximas eficiencias publicadas, 68\% [15] y 60\% [18] bombeando en las bandas de $790 \mathrm{~nm}$ y $1600 \mathrm{~nm}$, respectivamente. Hay que tener en cuenta que en la cavidad se ha optimizado la longitud de la TDF, pero no la relación de acoplo. Se eligió una relación de acoplo alta para que la potencia de bombeo umbral fuese baja, ya que no se dispone de una potencia de bombeo elevada. Disminuyendo dicha relación, aunque la potencia de bombeo umbral aumentaría, la eficiencia del láser crecería considerablemente. También disminuiría la longitud de onda de emisión del láser. Con la relación de acoplo elegida y, como la cavidad no incluye ningún elemento selector de longitud de onda, el espectro de emisión de los láseres de TDF emite en dos longitudes de onda situadas en torno a 1910-1920 nm con una separación entre ambas de unos pocos nanómetros.

También se han llevado a cabo medidas de ganancia espectral, las cuales resultan útiles para explicar cualitativamente el comportamiento de los amplificadores y láseres de TDF. El rango espectral de amplificación y de sintonía del láser se estima superior a $300 \mathrm{~nm}$. Finalmente, se ha demostrado la utilidad de este tipo de amplificadores ópticos para mejorar el espectro de las fuentes de supercontinuo en la 
región espectral desde $1800 \mathrm{~nm}$ hasta $2000 \mathrm{~nm}$. Como la fuente de supercontinuo está pulsada, esta mejora permitiría bombear fibras ópticas altamente no lineales cuya longitud de onda de dispersión cero esté situada en dicha región espectral.

\section{Agradecimientos / Acknowledgements}

Los autores agradecen el apoyo de la Diputación General de Aragón para el grupo de investigación de referencia T20_17R Grupo de Tecnologías Fotónicas (GTF).

\section{Anexo: Fuente de espectro supercontinuo}

Como fuente óptica para las medidas de amplificación, atenuación y transmisión se ha empleado una fuente de supercontinuo construida en nuestro laboratorio [23], cuyo esquema se muestra en la Fig. 18. A grandes rasgos, consiste en un láser de fibra óptica dopada con erbio con cavidad de anillo y pulsado mediante una técnica pasiva de mode-locking basada en el efecto de rotación no lineal de la polarización (nonlinear polarization rotation, NPR). El modulador pasivo está formado por un polarizador lineal situado entre dos controladores de polarización (modelo Polarite de General Photonics). Como medio activo del láser pulsado se emplea un EDFA que opera en banda $C$ con una potencia de saturación de salida de $+20 \mathrm{dBm}$ (Highwave, model C20-G20-H-FC/APC-BTO 3.0). El tren de pulsos generado se amplifica mediante un segundo EDFA colocado fuera de la cavidad de anillo, el cual también opera en banda $\mathrm{C}$ con una potencia de saturación de salida de $+26 \mathrm{dBm}$ (Manlight, model HWT-EDFA-GM-SC-BO-C26). Se emplean $25 \mathrm{~m}$ de fibra altamente no lineal (Yangtze Optical Fibre and Cable Company Ltd., tipo NL-1550Zero), la cual ofrece una dispersión prácticamente nula en $1550 \mathrm{~nm}$, con una pendiente en la dispersión menor que 0.025 ps nm-2 km-1. Su coeficiente no lineal es mayor que $10 \mathrm{~W}_{-1} \mathrm{~km}-1$ y su coeficiente de ganancia Raman es superior a $4.8 \mathrm{~W}-1 \mathrm{~km}-1$. Para mejorar la potencia espectral del supercontinuo se incluyen dentro de la cavidad $111 \mathrm{~m}$ de fibra óptica de dispersión positiva (Corning, modelo SMF28e) y fuera de la cavidad se colocan $22 \mathrm{~m}$ de fibra óptica de dispersión negativa (Thorlabs, modelo DCF38). Se consigue un tren de pulsos de 0.18 ps de duración con una frecuencia de repetición de 1.4 MHz. Dicho tren de pulsos se amplifica mediante un amplificador de fibra óptica dopada con erbio y bombea una muestra de fibra óptica de sílice altamente no lineal (highly nonlinear fiber, HNLF), produciendo el ensanchamiento del espectro desde $1100 \mathrm{~nm}$ hasta $2300 \mathrm{~nm}$, aproximadamente. Los espectros del tren de pulsos amplificado y del supercontinuo generado se muestran en la Fig. 19.

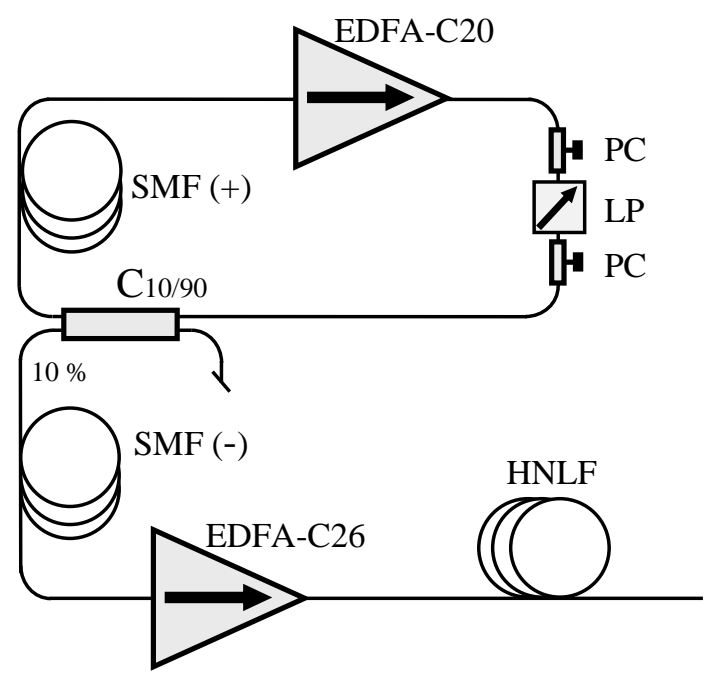

Fig.18. Esquema de la fuente de supercontinuo. PC: controlador de polarización, LP: polarizador lineal, C: acoplador, EDFA-C20/C26: amplificador de fibra dopada con erbio operando en banda C con potencia de saturación de salida de 20/26 dBm, SMF(+/-): fibra óptica monomodo con dispersión positiva/negativa, HNLF: fibra altamente no lineal 
ÓPTICA PURA Y APLICADA

www.sedoptica.es

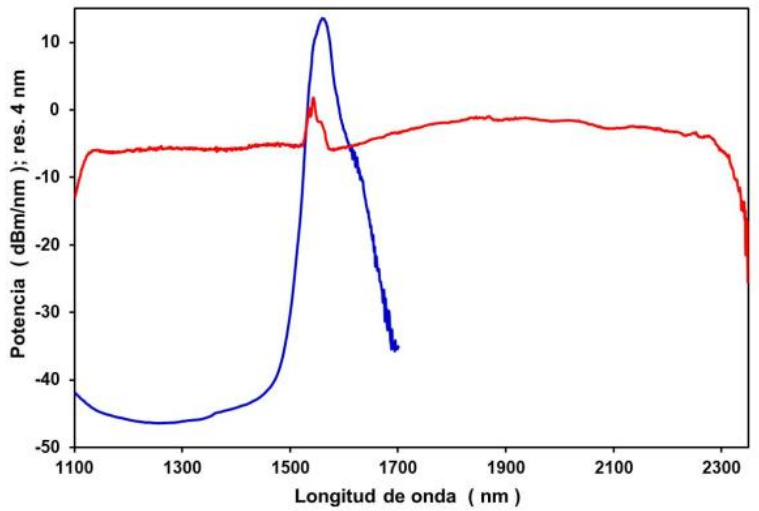

Fig.19. Espectros del tren de pulsos de bombeo (línea azul) y del supercontinuo generado (línea roja). 